\title{
Periodontal disease, preterm birth and low birthweight
}

\author{
Does periodontal disease in pregnant mothers increase the risk of preterm birth \\ or low birthweight?
}

\begin{abstract}
Khader YS, Ta'ani Q. Periodontal diseases and the risk of preterm birth and low birth weight: a meta-analysis. J Periodontol 2005; 76:161-165

Data sources Medline was searched for studies from 1966 to August 2002; reference lists from those identified were also used to find other articles.

Study selection Inclusion criteria specified observational studies that provided a risk estimate of preterm birth (PTB) or preterm low birthweight (PLBW) in babies of mothers who had periodontal diseases. Cases were defined as PTB if delivery was at $<37$ weeks' gestation, or as PLBW if an infant at delivery had a birthweight $<2500 \mathrm{~g}$ and one or more of the following: gestational age $<37$ weeks, preterm labour or premature rupture of membrane. Studies should have defined the exposure as periodontal disease and exposure should have been used as a categorical variable in the multivariate analysis. Articles were limited to those published in English and conducted on a human study population.
\end{abstract}

Data extraction and synthesis The strength of the relationship between periodontal disease and the risk of PTB was assessed in these studies by relative risk (RR) or odds ratio (OR). Because the risk of preterm birth was low in these studies, the relative odds provided a good approximation of RR. OR was obtained directly from each study or calculated using the available data. Before pooling the data, OR from individual studies were transformed to their natural logarithms $[\log (\mathrm{OR})]$ to stabilise the variances and normalise the distributions. For calculation of the pooled OR, each study was assigned a weight equalling the reciprocal of the variance of the $\log (\mathrm{OR})$ in that study. When the variances of $\log (\mathrm{OR}) \mathrm{s}$ were not reported directly in manuscripts, they were calculated. Estimates of the overall OR and the corresponding $95 \%$ confidence intervals $(\mathrm{Cl})$ were calculated using both fixed-effects and random-effects models. Homogeneity of effectsize across studies was tested by $Q$ statistics. A sensitivity analysis was conducted to assess the robustness of our findings for different exclusion criteria.

Results Five studies (three cohort and two case-control) were included. Pregnant women with periodontal disease had an overall adjusted risk of PTB that was 4.28 -fold $(95 \% \mathrm{Cl}, 2.62-6.99 ; P<0.005)$ the risk for subjects who did not have periodontal disease. The overall adjusted OR of PLBW was 5.28 (95\% Cl, 2.21-12.62; $P<0.005)$, whereas the overall adjusted OR of a delivery of either PTB or PLBW was $2.30(95 \% \mathrm{Cl}, 1.21-4.38 ; P<0.005)$.

Conclusions The findings indicate that periodontal diseases in the pregnant mother significantly increase the risk of subsequent PTB or PLBW. Although it remains important to promote good oral hygiene during routine prenatal visits, there is no convincing evidence, on the basis of existing case-control and prospective studies, that treatment of periodontal disease will reduce the risk of PTB. Consequently, large, randomised, placebo-controlled, masked clinical trials are required.

Address for correspondence: Yousef $S$ Khader, Department of Community Medicine, Public Health and Family Medicine, Faculty of Medicine, Jordan University of Science and Technology, Irbid 22110, Jordan. E-mail: yousef.k@excite.com or ykhader@tulane.edu.

\section{Commentary}

PTB and PLBW are serious complications in neonatology, representing a major problem for the families involved and a significant burden for any healthcare system. ${ }^{1}$ The list of risk factors reported for those poor pregnancy outcomes is large and includes: maternal age, parity, pre- and antenatal care, socioeconomic factors, marital status, infant gender, smoking, previous PTB/PLBW delivery, interpregnancy interval, nutritional status, drug abuse, maternal systemic inflammation, cervical incompetence, placenta previa, diabetes and stress. Uncovering the existence of modifiable risk factors for those outcomes is relevant.

In this review, the authors attempt to summarise the available evidence from observational studies on the association between periodontal diseases and PTB/PLBW. The studies included were considered to be of poor to fair quality and there was considerable variation in the definition of exposures and outcomes across the studies. Nevertheless, " ... the temptation to combine the results of studies seems to be hard to resist" ${ }^{\prime 2}$ and a meta-analysis of casecontrol and cohort studies was conducted.

Meta-analyses of observational studies are becoming popular in the biomedical literature ${ }^{3}$ and dentistry will probably witness many more in the near future. The main contribution of those metaanalyses is to reveal the amount and sources of heterogeneity of different studies ${ }^{2}$ and not to obtain a single summary estimate for the associations. If summary estimates are reported, they should be interpreted cautiously because observational studies can be seriously influenced by confounding and bias: this type of metaanalysis can produce very precise but equally spurious results. ${ }^{2,4} \mathrm{~A}$ limitation difficult to circumvent is that these analyses usually combine average estimates from studies designed to answer different questions in different populations.

In this review, the authors used quality scoring for the evaluation of the studies, merging information in a way that can obscure sources of heterogeneity, bias and confounding. ${ }^{4-6}$ Because of the small number of studies included, sensitivity analysis and the use of funnel plots are of little help investigating heterogeneity and are insufficient to assess the impact of confounding and selection bias.

Regarding confounding, several risk factors are common for the exposure and outcomes investigated; meanwhile, the covariates representing confounding variables are not common across the studies, which makes it impossible to adjust for confounding in the review. Furthermore, the confounding effect of socioeconomic variation which, according to the authors, is one of the main risk factors for the pregnancy outcomes investigated was broadly ignored in the studies that were included in the review.

There is also the possibility of bias. The authors do not provide information from the studies, which were categorised as of poor to fair quality, about the quality of blinding of the examiners, the selection of the study participants or the comparability of the study groups. It is not feasible, therefore, to assess the extent to which the results reported could arise from selection or information bias. The authors included case-control and prospective studies without discussing the possible implications of pooling results taken from 
different study designs. Finally, observational studies with positive associations are more likely to be published than those studies reporting no associations. Although the role of this publication bias may be evaluated to some extent for randomised controlled trials (RCT) using currently established RCT registers, it remains obscure how many observational studies remain unpublished because of their negative findings. The small number of studies included in this review makes it difficult to interpret the results of the plot reported as indicative of lack of publication bias.

The authors conclude, "Our findings indicate that periodontal diseases in the pregnant mother significantly increase the risk of subsequent preterm birth or low birth weight" and "large randomized placebo-control, masked clinical trials are required". These conclusions are not commensurate with the findings of the study, however.

The results of this review suggest that appropriate evidence is not available from good-quality observational research that could support the association between periodontal disease and PTB/ PLBW. This seems to be confirmed by two relatively wellconducted large observational studies ${ }^{7,8}$ whose results discourage the initiation of resource draining and potentially hazardous intervention studies on this issue. Future attempts to conduct systematic reviews of observational studies should make use of the available guidelines for reporting. ${ }^{3}$ The notion that the statistical combination of data should not be a prominent component of this type of review ${ }^{2}$ should also be taken into account.

\section{Practice point}

- There is insufficient evidence from observational research to support the association between periodontal diseases and preterm birth and/or low birthweight.

\section{Rodrigo López}

Department of Community Oral Health and Paediatric Dentistry, Faculty of Health Sciences, University of Aarhus, Aarhus, Denmark

1. Petrou S, Sach T, Davidson L. The long-term costs of preterm birth and low birth weight: results of a systematic review. Child Care Health Dev 2001; 27:97-115.

2. Egger M, Schneider M, Smith GD. Meta-analysis spurious precision? Meta-analysis of observational studies. Br Med J 1998; 316:140-144.

3. Stroup DF, Berlin JA, Morton SC, et al. Meta-analysis of observational studies in epidemiology: a proposal for reporting. Meta-analysis of observational studies in epidemiology (MOOSE) group. J Am Med Assoc 2000; 283:2008-2012.

4. Shapiro S. Meta-analysis/Shmeta-analysis. Am J Epidemiol 1994; 140:771-778.

5. Greenland S. Invited commentary: a critical look at some popular meta-analytic methods. Am J Epidemiol 1994; 140:290-296.

6. Greenland S. Can meta-analysis be salvaged? Am J Epidemiol 1994; 140:783-787.

7. Moore S, Ide M, Coward PY, et al. A prospective study to investigate the relationship between periodontal disease and adverse pregnancy outcome. B Dent | 2004; 197:251-258.

8. Davenport ES, Williams CECS, Sterne JAC, Murad S, Sivapathasundaram V, Curtis MA. Maternal periodontal disease and preterm low birthweight: case-control study. J Dent Res 2002; 81:313-318.

Evidence-Based Dentistry (2005) 6, 90-91.

doi:10.1038/sj.ebd.6400361 